\title{
STABILITY RESULT FOR A WEAKLY NONLINEARLY DAMPED POROUS SYSTEM WITH DISTRIBUTED DELAY
}

\section{KHOUDIR KIBECHE ${ }^{1}$, LAMINE BOUZETTOUTA ${ }^{2, *}$, ABDELHAK DJEBABLA $^{3}$, FAHIMA HEBHOUB ${ }^{2}$}

\author{
${ }^{1}$ University of Badji Mokhtar, Annaba, Algeria \\ ${ }^{2}$ Laboratory Applied Mathematics and History and Didactics of Mathematic, University 20 August 1955, \\ Skikda, Algeria \\ ${ }^{3}$ Laboratory of Applied Mathematics, Badji Mokhtar University-Annaba, P.O. Box 12, 23000 Annaba, \\ Algeria
}

*Corresponding author: lami_750000@yahoo.fr, 1.bouzettouta@univ-skikda.dz

\begin{abstract}
In this paper, we consider a one-dimensional porous system damped with a single weakly nonlinear feedback and distributed delay term. Without imposing any restrictive growth assumption near the origin on the damping term, we establish an explicit and general decay rate, using a multiplier method and some properties of convex functions in case of the same speed of propagation in the two equations of the system. The result is new and opens more research areas into porous-elastic system.
\end{abstract}

Received June $5^{\text {th }}, 2021$; accepted June $30^{\text {th }}, 2021$; published October $28^{\text {th }}, 2021$.

2010 Mathematics Subject Classification. 35B35, 35B40, 93D20.

Key words and phrases. porous system; general decay; nonlinear damping; distributed delay.

(C)2021 Authors retain the copyrights of their papers, and all open access articles are distributed under the terms of the Creative Commons Attribution License. 


\section{IntroduCtion}

In this paper, we consider the following porous system:

$$
\left\{\begin{array}{lc}
\rho u_{t t}-\mu u_{x x}-b \phi_{x}+\mu_{1} u_{t}+\int_{\tau_{1}}^{\tau_{2}} \mu_{2}(s) u_{t}(x, t-s) d s=0, x \in(0,1), t>0 \\
j \phi_{t t}-\delta \phi_{x x}+b u_{x}+\xi \phi+\alpha(t) g\left(\phi_{t}\right)=0, & x \in(0,1), t>0 \\
u(x, 0)=u_{0}(x), u_{t}(x, 0)=u_{1}(x), & x \in(0,1), \\
\phi(x, 0)=\phi_{0}(x), \phi_{t}(x, 0)=\phi_{1}(x), & x \in(0,1), \\
u_{x}(0, t)=u_{x}(1, t), \phi(0, t)=\phi(1, t)=0 & \\
u_{t}(x,-t)=f_{0}(x, t) & \text { in } \quad(0,1) \times\left(0, \tau_{2}\right)
\end{array}\right.
$$

Firstly, to deal with the delay term, we introduce the new variable [17]

$$
z(x, \rho, s, t)=u_{t}(x, t-\rho s), x \in(0,1), \rho \in(0,1), \rho \in\left(\tau_{1}, \tau_{2}\right), t>0
$$

Then we obtain

$$
s z_{t}(x, \rho, s, t)+z_{\rho}(x, \rho, s, t)=0, x \in(0,1), \rho \in(0,1), \rho \in\left(\tau_{1}, \tau_{2}\right), t>0
$$

Then problem (1.1) is equivalent to

$$
\left\{\begin{array}{lc}
\rho u_{t t}-\mu u_{x x}-b \phi_{x}+\mu_{1} u_{t}+\int_{\tau_{1}}^{\tau_{2}} \mu_{2}(s) z(x, 1, t, s) d s=0, & x \in(0,1), t>0, \\
j \phi_{t t}-\delta \phi_{x x}+b u_{x}+\xi \phi+\alpha(t) g\left(\phi_{t}\right)=0, & x \in(0,1), t>0, \\
s z_{t}(x, \rho, s, t)+z_{\rho}(x, \rho, s, t)=0, x \in(0,1), \rho \in(0,1), \rho \in\left(\tau_{1}, \tau_{2}\right), t>0 \\
u(x, 0)=u_{0}(x), u_{t}(x, 0)=u_{1}(x), & x \in(0,1), \\
\phi(x, 0)=\phi_{0}(x), \phi_{t}(x, 0)=\phi_{1}(x), & x \in(0,1), \\
u_{x}(0, t)=u_{x}(1, t), \phi(0, t)=\phi(1, t)=0 & (x, \rho, s) \in(0,1) \times(0,1) \times\left(\tau_{1}, \tau_{2}\right)
\end{array}\right.
$$

In recent paper, Apalara in [2] considered the following on-dimensional porous system damped with a single weakly nonlinear feedback

$$
\begin{cases}\rho u_{t t}-\mu u_{x x}-b \phi_{x}=0, x \in(0,1), t>0, & \\ j \phi_{t t}-\delta \phi_{x x}+b u_{x}+\xi \phi+\alpha(t) g\left(\phi_{t}\right)=0, & x \in(0,1), t>0 \\ u(x, 0)=u_{0}(x), u_{t}(x, 0)=u_{1}(x), & x \in(0,1), \\ \phi(x, 0)=\phi_{0}(x), \phi_{t}(x, 0)=\phi_{1}(x), & x \in(0,1) \\ u_{x}(0, t)=u_{x}(1, t), \phi(0, t)=\phi(1, t)=0 & \end{cases}
$$

Without in pasing an explicit and general decay rate, he used a multiplier method and some proprieties of convex functions in case of the same speed of propagation in the both equation of the system. The same author, in [3] considered a porous-elastic system with memory term acting only on the porous equation, with 
the mixed boundary Neumann-Direchlet conditions, he proved a general decay result, for which exponential and polynomial decay results are special cases.

Back to system (1.1), it is to be noted that when $\mu_{1}=\mu_{2}=0$ and replacing the term $\alpha(t) g\left(\phi_{t}\right)$ by the term $\int_{0}^{t} g(t-s) u_{x x}(x, s) d s$ then (1.1) is equivalent to the well-known Timoshenko system of memory type which is exponentially stable depending of the relaxation function $g$ and provided that the wave speeds of the system are equal (See $[1,15])$.

Messaoudi and Fareh [16] investigated the following system:

$$
\left\{\begin{array}{l}
\rho u_{t t}=\mu u_{x x}+b \phi_{x}-\beta \theta_{x}, \text { in }(0,1) \times(0, \infty), \\
j \phi_{t t}=\alpha \phi_{x x}-b u_{x}+\xi \phi+m \theta+\tau \phi_{t}, \quad \text { in }(0,1) \times(0, \infty), \\
c \phi_{t}=-q_{x}-\beta u_{t x}-m \phi_{t}, \quad \text { in }(0,1) \times(0, \infty), \\
\tau_{0} q_{t}-q+k \theta_{x}=0, \quad \text { in }(0,1) \times(0, \infty),
\end{array}\right.
$$

and established, using the energy method, an exponential decay result. For more results on the subject, we refer the reader to $[5,10,11,19]$.

Concerning the weight of the delay, we assume that

$$
\int_{\tau_{1}}^{\tau_{2}}\left|\mu_{2}(s)\right| d s<\mu_{1}
$$

and establish the well-posedness as well as the exponential stability results of the energy $E(t)$, defined by

$$
\begin{aligned}
E(t)= & \frac{1}{2} \int_{0}^{1}\left[\rho u_{t}^{2}+\mu u_{x}^{2}+\xi \phi^{2}+\delta \phi_{x}^{2}+j \phi_{t}^{2}+2 b \phi u_{x}\right] d x \\
& +\frac{1}{2} \int_{0}^{1} \int_{0}^{1} \int_{\tau_{1}}^{\tau_{2}} s\left|\mu_{2}(s)\right| z^{2}(x, \rho, s, t) d s d \rho d x
\end{aligned}
$$

\section{Preliminaries}

In this section, we present some materials needed in the proof of our result. We assume $\alpha$ and $g$ satisfy the following hypotheses:

(H1) $\quad \alpha: \mathbb{R}^{+} \rightarrow \mathbb{R}_{*}^{+}$is a non-increasing differentiable function;

(H2) $g: \mathbb{R} \rightarrow \mathbb{R}$ is a non-decreasing $C^{0}$-function such that there exist positive constants $c_{1}, c_{2}, \eta$ and $G \in C^{1}([0, \infty))$, with $G(0)=0$, and $G$ is linear or strictly convex $C^{2}$-function on $(0, \eta]$ such that

$$
\left\{\begin{array}{l}
s^{2}+g^{2}(s) \leq G^{-1}(s g(s)) \text { for all }|s| \leq \eta \\
c_{1}|s| \leq|g(s)| \leq c_{2}|s| \text { for all }|s| \geq \eta
\end{array}\right.
$$

Remark 2.1. Hypothesis (H2) implies that $s g(s)>0$ for all $s \neq 0$.

* According to our knowledge, hypothesis (H2) with $\eta=1$ was first introduced by Lasiecka and Tataru [13]. They established a decay result, which depends on the solution of an explicit nonlinear ordinary differential equation. Furthermore, they proved that the monotonicity and continuity of g guarantee the existence of the function $G$ defined in (H2). 
For completeness purpose we state, without proof, the existence and regularity result of system (1.1). First, we introduce the following spaces:

$$
\mathcal{H}=H_{*}^{1}(0,1) \times L_{*}^{2}(0,1) \times H^{1}(0,1) \times L^{2}(0,1) \times L^{2}\left((0,1) \times(0,1) \times\left(\tau_{1}, \tau_{2}\right)\right)
$$

and

$$
\begin{aligned}
\widetilde{\mathcal{H}}= & \phi_{0} \in\left[H_{*}^{2}(0,1) \cap H_{*}^{1}(0,1)\right] \times H_{*}^{1}(0,1) \times\left[H^{2}(0,1) \cap H^{1}(0,1)\right] \\
& \times H^{1}(0,1) \times L^{2}\left((0,1) \times(0,1) \times\left(\tau_{1}, \tau_{2}\right)\right)
\end{aligned}
$$

where

$$
\begin{gathered}
L_{*}^{2}(0,1)=\left\{\psi \in L^{2}(0,1): \int_{0}^{1} \psi(x) d x=0\right\}, \\
H_{*}^{1}(0,1)=H^{1}(0,1) \times L_{*}^{2}(0,1), \\
H_{*}^{2}(0,1)=\left\{\psi \in H^{2}(0,1): \psi_{x}(0)=\psi_{x}(1)=0\right\} .
\end{gathered}
$$

For $U=\left(u, u_{t}, \phi, \phi_{t}, z\right)$, we have the following existence and regularity result:

Proposition 2.1. Assume that (H1) and (H2) are satisfied. Then for all $U_{0} \in \mathcal{H}$, the system (1.1) has a unique global (weak) solution

$$
u \in C\left(\mathbb{R}_{+} ; H_{*}^{1}(0,1)\right) \cap C^{1}\left(\mathbb{R}_{+} ; L_{*}^{2}(0,1)\right), \phi \in C\left(\mathbb{R}_{+} ; H^{1}(0,1)\right) \cap C^{1}\left(\mathbb{R}_{+} ; L^{2}(0,1)\right) .
$$

Moreover, if $U_{0} \in \widetilde{\mathcal{H}}$, then the solution satisfies

$$
\begin{aligned}
& u \in L^{\infty}\left(\mathbb{R}_{+} ; H_{*}^{2}(0,1) \cap H_{*}^{1}(0,1)\right) \cap W^{1, \infty}\left(\mathbb{R}_{+} ; H_{*}^{1}(0,1)\right) \cap W^{2, \infty}\left(\mathbb{R}_{+} ; L_{*}^{2}(0,1)\right) \\
& \phi \in L^{\infty}\left(\mathbb{R}_{+} ; H^{2}(0,1) \cap H_{0}^{1}(0,1)\right) \cap W^{1, \infty}\left(\mathbb{R}_{+} ; H_{0}^{1}(0,1)\right) \cap W^{2, \infty}\left(\mathbb{R}_{+} ; L^{2}(0,1)\right)
\end{aligned}
$$

Remark 2.2. This result can be proved using the theory of maximal nonlinear monotone operators (see [8]).

\section{Technical Lemmas}

In this section, we state and prove our stability results for the energy of system (1.1) by using the multiplier technique. To achieve our goal, we need the following lemmas.

Lemma 3.1. Let $(u, \phi, z)$ be the solution of(1.2), then we have

$$
E^{\prime}(t) \leq-m_{e} \int_{0}^{1} u_{t}^{2} d x-\int_{0}^{1} \alpha(t) \phi_{t} g\left(\phi_{t}\right) d x \leq 0
$$


Proof. Multiplying $(1.2)_{1}$, and $(1.2)_{2}$ by $u_{t}, \phi_{t}$ respectively, and integrating over $(0,1)$, using integration by parts and the boundary conditions, we obtain

$$
\begin{aligned}
& \frac{1}{2} \frac{d}{d t} \int_{0}^{1}\left(\rho u_{t}^{2}+\mu u_{x}^{2}+\xi \phi^{2}+\delta \phi_{x}^{2}+j \phi_{t}^{2}+2 b \phi u_{x}\right) d x= \\
& -\int_{0}^{1} \alpha(t) \phi_{t} g\left(\phi_{t}\right) d x-\mu_{1} \int_{0}^{1} u_{t}^{2} d x-\int_{0}^{1} u_{t} \int_{\tau_{1}}^{\tau_{2}} \mu_{2}(s) z(x, 1, t, s) d s d x
\end{aligned}
$$

Multiplying $(1.2)_{3}$ by $\left|\mu_{2}(s)\right| z$, integrating the product over $(0,1) \times(0,1) \times\left(\tau_{1}, \tau_{2}\right)$, and recall that $z(x, 0, s, t)=$ $u_{t}$, we get

$$
\begin{aligned}
\frac{1}{2} \frac{d}{d t} \int_{0}^{1} \int_{0}^{1} \int_{\tau_{1}}^{\tau_{2}} s\left|\mu_{2}(s)\right| z^{2}(x, \rho, t, s) d s d \rho d x= & -\frac{1}{2} \int_{0}^{1} \int_{\tau_{1}}^{\tau_{2}}\left|\mu_{2}(s)\right| z^{2}(x, 1, t, s) d s d x \\
& +\frac{1}{2} \int_{0}^{1} u_{t} \int_{\tau_{1}}^{\tau_{2}}\left|\mu_{2}(s)\right| d s d x
\end{aligned}
$$

A combination of (3.2) and (3.3) gives

$$
E^{\prime}(t)=-\int_{0}^{1} \alpha(t) \phi_{t} g\left(\phi_{t}\right) d x-\mu_{1} \int_{0}^{1} u_{t}^{2} d x-\int_{0}^{1} u_{t} \int_{\tau_{1}}^{\tau_{2}} \mu_{2}(s) z(x, 1, t, s) d s d x
$$

with

$$
-\int_{0}^{1} u_{t} \int_{\tau_{1}}^{\tau_{2}} \mu_{2}(s) z(x, 1, t, s) d s d x \leq \frac{1}{2} \int_{\tau_{1}}^{\tau_{2}}\left|\mu_{2}(s)\right| \int_{0}^{1} u_{t}^{2} d x+\frac{1}{2} \int_{0}^{1} \int_{\tau_{1}}^{\tau_{2}}\left|\mu_{2}(s)\right| z^{2}(x, 1, t, s) d s d x
$$

then

$$
E^{\prime}(t) \leq-\int_{0}^{1} \alpha(t) \phi_{t} g\left(\phi_{t}\right) d x-\left(\mu_{1}-\int_{\tau_{1}}^{\tau_{2}}\left|\mu_{2}(s)\right|\right) \int_{0}^{1} u_{t}^{2} d x
$$

taking $\left(\mu_{1}-\int_{\tau_{1}}^{\tau_{2}}\left|\mu_{2}(s)\right|\right)=m_{e}$ we obtain $(3.1)$.

Lemma 3.2. Assume that (H1) and (H2) hold. Then, for all $U_{0} \in \mathcal{H}$, the functional

$$
F_{1}(t)=j \int_{0}^{1} \phi_{t} \phi d x+\frac{b \rho}{\mu} \int_{0}^{1} \phi \int_{0}^{x} u_{t}(y) d y d x
$$

satisfies, for any $\varepsilon_{1}>0$

$$
\begin{aligned}
F_{1}^{\prime}(t) \leq & \left(j+\frac{\varepsilon_{1} b \rho}{\mu}\right) \int_{0}^{1} \phi_{t}^{2} d x-j \delta \int_{0}^{1} \phi_{x}^{2} d x+b j \varepsilon_{1} \int_{0}^{1} u_{x}^{2} d x+\frac{b \rho}{4 \varepsilon_{1} \mu} \int_{0}^{1} u_{t}^{2} d x \\
& +\left(j \alpha(0) \varepsilon_{1}+\frac{b j}{4 \varepsilon_{1}}-\xi j\right) \int_{0}^{1} \phi^{2} d x+\frac{j \alpha(0)}{4 \varepsilon_{1}} \int_{0}^{1} g^{2}\left(\phi_{t}\right) d x
\end{aligned}
$$

Proof. Differentiating $F_{1}(t)$, taking into account (1.2) using integrating by parts, and Young's inequality, we obtain

$$
\begin{aligned}
F_{1}^{\prime}(t) \leq & j \int_{0}^{1} \phi_{t}^{2} d x-j \delta \int_{0}^{1} \phi_{x}^{2} d x+b j \varepsilon_{1} \int_{0}^{1} u_{x}^{2} d x+\frac{c_{p} b j}{4 \varepsilon_{1}} \int_{0}^{1} \phi_{x}^{2} d x-\xi j c_{p} \int_{0}^{1} \phi_{x}^{2} d x \\
& -j \int_{0}^{1} \alpha(t) \phi g\left(\phi_{t}\right) d x+\frac{b \rho}{\mu} \int_{0}^{1} \phi_{t} \int_{0}^{x} u_{t}(y) d y d x \\
& +\frac{b \rho}{\mu} \int_{0}^{1} \phi \frac{d}{d t}\left(\int_{0}^{x} u_{t}(y) d y\right) d x
\end{aligned}
$$


By Caucy-Schwartz inequality, it is clear that

$$
\int_{0}^{1}\left(\int_{0}^{x} u_{t}(y) d y\right)^{2} d x \leq \int_{0}^{1}\left(\int_{0}^{1} u_{t} d x\right)^{2} d x \leq \int_{0}^{1} u_{t}^{2} d x
$$

then

$$
\begin{aligned}
F_{1}^{\prime}(t) \leq & j \int_{0}^{1} \phi_{t}^{2} d x-j \delta \int_{0}^{1} \phi_{x}^{2} d x+b j \varepsilon_{1} \int_{0}^{1} u_{x}^{2} d x+\frac{c_{p} b j}{4 \varepsilon_{1}} \int_{0}^{1} \phi_{x}^{2} d x-\xi j c_{p} \int_{0}^{1} \phi_{x}^{2} d x \\
& -j \int_{0}^{1} \alpha(t) \phi g\left(\phi_{t}\right) d x \\
& +\frac{\varepsilon_{1} b \rho}{\mu} \int_{0}^{1} \phi_{t}^{2} d x+\frac{b \rho}{4 \varepsilon_{1} \mu} \int_{0}^{1}\left(\int_{0}^{x} u_{t}(y) d y\right)^{2} d x \\
& +\frac{b \rho}{\mu} \int_{0}^{1} \phi \frac{d}{d t}\left(\int_{0}^{x} u_{t}(y) d y\right) d x
\end{aligned}
$$

thus we obtain

$$
\begin{aligned}
F_{1}^{\prime}(t) \leq & \left(j+\frac{\varepsilon_{1} b \rho}{\mu}\right) \int_{0}^{1} \phi_{t}^{2} d x-j \delta \int_{0}^{1} \phi_{x}^{2} d x+b j \varepsilon_{1} \int_{0}^{1} u_{x}^{2} d x+\frac{b \rho}{4 \varepsilon_{1} \mu} \int_{0}^{1} u_{t}^{2} d x \\
& +\left(j \alpha(t) \varepsilon_{1}+\frac{b j}{4 \varepsilon_{1}}-\xi j\right) \int_{0}^{1} \phi^{2} d x+\frac{j \alpha(t)}{4 \varepsilon_{1}} \int_{0}^{1} g^{2}\left(\phi_{t}\right) d x
\end{aligned}
$$

Lemma 3.3. Assume that (H1), (H2) and (3.8) hold. Then, for all $U_{0} \in \mathcal{H}$, the functional

$$
F_{2}(t)=b \int_{0}^{1} \phi_{x} u_{t} d x+b \int_{0}^{1} \phi_{t} u_{x} d x
$$

satisfies, for any $\varepsilon_{2}>0$

$$
\begin{aligned}
F_{2}^{\prime}(t) \leq & \left(\frac{b^{2}}{\rho}+\varepsilon_{2} \frac{b \mu_{1}}{\rho}+\frac{b n_{0}}{2 \rho}\right) \int_{0}^{1} \phi_{x}^{2} d x-\left(\frac{b^{2}}{j}-\frac{b \xi}{4 \varepsilon_{2} j}-\frac{b}{j} \alpha(t)\right) \int_{0}^{1} u_{x}^{2} d x \\
& +\frac{b \mu_{1}}{4 \varepsilon_{2} \rho} \int_{0}^{1} u_{t}^{2} d x+\varepsilon_{2} \frac{b \xi}{j} \int_{0}^{1} \phi^{2} d x+\frac{b}{j} \alpha(t) \int_{0}^{1} g^{2}\left(\phi_{t}\right) d x \\
& +\frac{1}{2} \frac{b}{\rho} \int_{0}^{1} \int_{\tau_{1}}^{\tau_{2}}\left|\mu_{2}(s)\right| z^{2}(x, 1, s, t) d s d x
\end{aligned}
$$

Proof. Simple computaions give

$$
\begin{aligned}
F_{2}^{\prime}(t)= & \frac{b^{2}}{\rho} \int_{0}^{1} \phi_{x}^{2} d x-\frac{b^{2}}{j} \int_{0}^{1} u_{x}^{2} d x \\
& +\frac{b \mu}{\rho} \int_{0}^{1} u_{x x} \phi_{x} d x-\frac{b \mu_{1}}{\rho} \int_{0}^{1} \phi_{x} u_{t} d x \\
& +\frac{b \delta}{j} \int_{0}^{1} \phi_{x x} u_{x} d x-\frac{b \xi}{j} \int_{0}^{1} \phi u_{x} d x \\
& -\frac{b}{\rho} \int_{0}^{1} \phi_{x} \int_{\tau_{1}}^{\tau_{2}} \mu_{2}(s) u_{t}(x, 1, t, s) d s d x-\frac{b}{j} \int_{0}^{1} \alpha(t) u_{x} g\left(\phi_{t}\right) d x
\end{aligned}
$$


taking into account the fact that

$$
\frac{\mu}{\rho}=\frac{\delta}{j}
$$

and using young's inequality

$$
\begin{aligned}
F_{2}^{\prime}(t) \leq & \left(\frac{b^{2}}{\rho}+\varepsilon_{2} \frac{b \mu_{1}}{\rho}\right) \int_{0}^{1} \phi_{x}^{2} d x+\left(\frac{b \xi}{4 \varepsilon_{2} j}-\frac{b^{2}}{j}+\frac{b}{j} \alpha(t)\right) \int_{0}^{1} u_{x}^{2} d x \\
& +\frac{b \mu_{1}}{4 \varepsilon_{2} \rho} \int_{0}^{1} u_{t}^{2} d x+\varepsilon_{2} \frac{b \xi}{j} \int_{0}^{1} \phi^{2} d x+\frac{b}{j} \alpha(t) \int_{0}^{1} g^{2}\left(\phi_{t}\right) d x \\
& -\frac{b}{\rho} \int_{0}^{1} \phi_{x} \int_{\tau_{1}}^{\tau_{2}} \mu_{2}(s) z(x, 1, t, s) d s d x \\
-\frac{b}{\rho} \int_{0}^{1} \phi_{x} \int_{\tau_{1}}^{\tau_{2}} \mu_{2}(s) z(x, 1, & t, s) d s d x \leq \frac{1}{2} \frac{b}{\rho} \int_{\tau_{1}}^{\tau_{2}}\left|\mu_{2}(s)\right| d s \int_{0}^{1} \phi_{x}^{2} d x+\frac{1}{2} \frac{b}{\rho} \int_{0}^{1} \int_{\tau_{1}}^{\tau_{2}}\left|\mu_{2}(s)\right| z^{2}(x, 1, s, t) d s d x \\
F_{2}^{\prime}(t) \leq \quad & \left(\frac{b^{2}}{\rho}+\varepsilon_{2} \frac{b \mu_{1}}{\rho}+\frac{b n_{0}}{2 \rho}\right) \int_{0}^{1} \phi_{x}^{2} d x-\left(\frac{b^{2}}{j}-\frac{b \xi}{4 \varepsilon_{2} j}-\frac{b}{j} \alpha(t)\right) \int_{0}^{1} u_{x}^{2} d x \\
& +\frac{b \mu_{1}}{4 \varepsilon_{2} \rho} \int_{0}^{1} u_{t}^{2} d x+\varepsilon_{2} \frac{b \xi}{j} \int_{0}^{1} \phi^{2} d x+\frac{b}{j} \alpha(t) \int_{0}^{1} g^{2}\left(\phi_{t}\right) d x \\
& +\frac{1}{2} \frac{b}{\rho} \int_{0}^{1} \int_{\tau_{1}}^{\tau_{2}}\left|\mu_{2}(s)\right| z^{2}(x, 1, s, t) d s d x
\end{aligned}
$$

with $\int_{\tau_{1}}^{\tau_{2}}\left|\mu_{2}(s)\right| d s=n_{0}$

Lemma 3.4. The functional

$$
F_{3}(t)=-\rho \int_{0}^{1} u_{t} u d x
$$

satisfies, for any $\varepsilon_{3}>0$

$$
\begin{aligned}
F_{3}^{\prime}(t)= & +\left(\mu+\frac{n_{0} c_{p}}{2}+c_{p} b \varepsilon_{3}\right) \int_{0}^{1} u_{x}^{2} d x \\
& +\frac{b}{4 \varepsilon_{3}} \int_{0}^{1} \phi_{x}^{2} d x-\left(\rho-\frac{\mu_{1}}{4 \varepsilon_{3}}\right) \int_{0}^{1} u_{t}^{2} d x \\
& +\frac{1}{2} \int_{0}^{1} \int_{\tau_{1}}^{\tau_{2}}\left|\mu_{2}(s)\right| z^{2}(x, 1, t, s) d s d x
\end{aligned}
$$

Proof. A simple differentiation of $F_{3}(t)$, using the first equation in (1.2), give

$$
\begin{aligned}
F_{3}^{\prime}(t)= & -\rho \int_{0}^{1} u_{t}^{2} d x+\mu \int_{0}^{1} u_{x}^{2} d x \\
& +c_{p} b \varepsilon_{3} \int_{0}^{1} u_{x}^{2} d x+\frac{b}{4 \varepsilon_{3}} \int_{0}^{1} \phi_{x}^{2} d x \\
& +\mu_{1} \varepsilon_{3} c_{p} \int_{0}^{1} u_{x}^{2} d x+\frac{\mu_{1}}{4 \varepsilon_{3}} \int_{0}^{1} u_{t}^{2} d x \\
& +\int_{0}^{1} \int_{\tau_{1}}^{\tau_{2}} \mu_{2}(s) u u_{t}(x, 1, t, s) d s d x \\
\int_{0}^{1} u \int_{\tau_{1}}^{\tau_{2}} \mu_{2}(s) u_{t}(x, 1, t, s) d s d x \leq & \frac{c_{p}}{2} \int_{\tau_{1}}^{\tau_{2}}\left|\mu_{2}(s)\right| \int_{0}^{1} u_{x}^{2} d x+\frac{1}{2} \int_{0}^{1} \int_{\tau_{1}}^{\tau_{2}}\left|\mu_{2}(s)\right| z^{2}(x, 1, t, s) d s d x
\end{aligned}
$$


then

$$
\begin{aligned}
F_{3}^{\prime}(t)= & +\left(\mu+\mu_{1} \varepsilon_{3} c_{p}+\frac{n_{0} c_{p}}{2}+c_{p} b \varepsilon_{3}\right) \int_{0}^{1} u_{x}^{2} d x \\
& +\frac{b}{4 \varepsilon_{3}} \int_{0}^{1} \phi_{x}^{2} d x-\left(\rho-\frac{\mu_{1}}{4 \varepsilon_{3}}\right) \int_{0}^{1} u_{t}^{2} d x \\
& +\frac{1}{2} \int_{0}^{1} \int_{\tau_{1}}^{\tau_{2}}\left|\mu_{2}(s)\right| z^{2}(x, 1, t, s) d s d x
\end{aligned}
$$

Lemma 3.5. The functional

$$
F_{4}(t)=\int_{0}^{1} \int_{0}^{1} \int_{\tau_{1}}^{\tau_{2}} s e^{-s \rho}\left|\mu_{2}(s)\right| z^{2}(x, \rho, t, s) d s d \rho d x
$$

satisfies, for some positive constant $m_{1}$, the following estimate

$$
\begin{aligned}
F_{4}^{\prime}(t) \leq & -m_{1} \int_{0}^{1} \int_{\tau_{1}}^{\tau_{2}}\left|\mu_{2}(s)\right| z^{2}(x, 1, t, s) d s d x+\int_{\tau_{1}}^{\tau_{2}}\left|\mu_{2}(s)\right| d s \int_{0}^{1} u_{t}^{2} d x \\
& -m_{1} \int_{0}^{1} \int_{0}^{1} \int_{\tau_{1}}^{\tau_{2}} s\left|\mu_{2}(s)\right| z^{2}(x, \rho, t, s) d s d \rho d x
\end{aligned}
$$

Proof. With

$$
\begin{aligned}
s z_{t}(x, \rho, t, s)+z_{\rho}(x, \rho, t, s) & =0 \text { in }(0,1) \times(0,1) \times\left(\tau_{1}, \tau_{2}\right) \times(0, \infty) \\
z_{t}(x, \rho, t, s) & =-\frac{1}{s} z_{\rho}(x, \rho, t, s)
\end{aligned}
$$

Differentiating $F_{4}(t)$, and using the equation (3.13), we obtain

$$
\begin{aligned}
F_{4}^{\prime}(t)= & 2 \int_{0}^{1} \int_{0}^{1} \int_{\tau_{1}}^{\tau_{2}} s e^{-s \rho}\left|\mu_{2}(s)\right| z z_{t}(x, \rho, t, s) d s d \rho d x \\
= & -\frac{\partial}{\partial \rho} \int_{0}^{1} \int_{0}^{1} \int_{\tau_{1}}^{\tau_{2}} e^{-s \rho}\left|\mu_{2}(s)\right| z^{2}(x, \rho, t, s) d s d \rho d x \\
& -\int_{0}^{1} \int_{0}^{1} \int_{\tau_{1}}^{\tau_{2}} s e^{-s \rho}\left|\mu_{2}(s)\right| z^{2}(x, \rho, t, s) d s d \rho d x \\
F_{4}^{\prime}(t)= & -\int_{0}^{1} \int_{\tau_{1}}^{\tau_{2}}\left|\mu_{2}(s)\right|\left[e^{-s \rho} z^{2}(x, 1, t, s)-z^{2}(x, 0, t, s)\right] d s d x \\
& -\int_{0}^{1} \int_{0}^{1} \int_{\tau_{1}}^{\tau_{2}} s e^{-s \rho}\left|\mu_{2}(s)\right| z^{2}(x, \rho, t, s) d s d \rho d x
\end{aligned}
$$

Using the fact that $z(x, 0, t, s)=u_{t}$ and $e^{-s} \leq e^{-s \rho} \leq 1$, for all $\rho \in[0,1]$, we obtain

$$
\begin{aligned}
F_{4}^{\prime}(t) \leq & -\int_{0}^{1} \int_{\tau_{1}}^{\tau_{2}}\left|\mu_{2}(s)\right| e^{-s \rho} z^{2}(x, 1, t, s) d s d x+\int_{\tau_{1}}^{\tau_{2}}\left|\mu_{2}(s)\right| d s \int_{0}^{1} u_{t}^{2} d x \\
& -\int_{0}^{1} \int_{0}^{1} \int_{\tau_{1}}^{\tau_{2}} s e^{-s \rho}\left|\mu_{2}(s)\right| z^{2}(x, \rho, t, s) d s d \rho d x
\end{aligned}
$$

Because $-s e^{-s}$ is an increasing function, we have $-s e^{-s} \leq-s e^{-\tau_{2}}$, for all $s \in\left[\tau_{1}, \tau_{2}\right]$ 
Finally, setting $m_{1}=e^{-\tau_{2}}$, with $\int_{\tau_{1}}^{\tau_{2}}\left|\mu_{2}(s)\right|<\mu_{1}$, we obtain

$$
\begin{aligned}
F_{4}^{\prime}(t) \leq & -m_{1} \int_{0}^{1} \int_{\tau_{1}}^{\tau_{2}}\left|\mu_{2}(s)\right| z^{2}(x, 1, t, s) d s d x+\int_{\tau_{1}}^{\tau_{2}}\left|\mu_{2}(s)\right| d s \int_{0}^{1} u_{t}^{2} d x \\
& -m_{1} \int_{0}^{1} \int_{0}^{1} \int_{\tau_{1}}^{\tau_{2}} s\left|\mu_{2}(s)\right| z^{2}(x, \rho, t, s) d s d \rho d x
\end{aligned}
$$

Lemma 3.6. Suppose (H1), (H2), and Eq. (3.8) hold. Let $U_{0} \in \mathcal{H}$. Then, for $N, N_{1}, N_{2}, N_{3}>0$ sufficiently large, the Lyapunov functional defined by

$$
\mathcal{L}(t):=N E(t)+N_{1} F_{1}(t)+N_{2} F_{2}(t)+F_{3}(t)+N_{3} F_{4}(t)
$$

satisfies, for some positive constants $d_{1}, d_{2}$ and $k_{1}$

$$
d_{1} \mathcal{L}(t) \leq E(t) \leq d_{2} \mathcal{L}(t), \forall t \geq 0
$$

and

$$
\mathcal{L}^{\prime}(t) \leq-k_{1} E(t)+c \int_{0}^{1}\left(\phi_{t}^{2}+g^{2}\left(\phi_{t}\right)\right) d x, \forall t \geq 0
$$

with

$$
\begin{aligned}
\mathcal{L}^{\prime}(t) \leq & {\left[\frac{b \rho}{4 \varepsilon_{1} \mu} N_{1}-N m_{e}+N_{3} \mu_{1}+\frac{b \mu_{1}}{4 \varepsilon_{2} \rho} N_{2}-\left(\rho-\frac{\mu_{1}}{4 \varepsilon_{3}}\right)\right] \int_{0}^{1} u_{t}^{2} d x } \\
& +\left(N_{1}\left(j+\frac{\varepsilon_{1} b \rho}{\mu}\right)\right) \int_{0}^{1} \phi_{t}^{2} d x \\
& +\left(b j \varepsilon_{1} N_{1}+\left(\mu+\frac{n_{0}}{2}+b \varepsilon_{3}\right)-N_{2}\left(\frac{b^{2}}{j}-\frac{b \xi}{4 \varepsilon_{2} j}-\frac{b}{j} \alpha(t)\right)\right) \int_{0}^{1} u_{x}^{2} d x \\
& +\left(N_{2} \frac{1}{2 \rho}\left(2 b^{2}+2 \varepsilon_{2} b \mu_{1}+b n_{0}\right)-j \delta N_{1}+\frac{b}{4 \varepsilon_{3}}\right) \int_{0}^{1} \phi_{x}^{2} d x \\
& +\left(\varepsilon_{2} \frac{b \xi}{j} N_{2}+N_{1}\left(j \alpha(t) \varepsilon_{1}+\frac{b j}{4 \varepsilon_{1}}-\xi j\right)\right) \int_{0}^{1} \phi^{2} d x \\
& +\left(N_{1} \frac{j \alpha(t)}{4 \varepsilon_{1}}+\frac{b}{j} \alpha(t) N_{2}\right) \int_{0}^{1} g^{2}\left(\phi_{t}\right) d x \\
& +\left(\frac{1}{2}\left(\frac{b N_{2}}{\rho}+1\right)-m_{1} N_{3}\right) \int_{0}^{1} \int_{\tau_{1}}^{\tau_{2}}\left|\mu_{2}(s)\right| z^{2}(x, 1, s, t) d s d x \\
& -m_{1} N_{3} \int_{0}^{1} \int_{0}^{1} \int_{\tau_{1}}^{\tau_{2}} s\left|\mu_{2}(s)\right| z^{2}(x, \rho, t, s) d s d \rho d x-N \int_{0}^{1} \alpha(t) \phi_{t} g\left(\phi_{t}\right) d x
\end{aligned}
$$

At this point, we have to choose our constants very carefully. First, choosing $\varepsilon_{3}<<1$, and $\varepsilon_{1}, \varepsilon_{2}$ small enough such that

$$
\varepsilon_{1} \leq \frac{b \rho N_{1}}{4 \mu\left(N m_{e}-N_{3} \mu_{1}\right)}, \varepsilon_{2} \leq \frac{b \mu_{1} N_{2}}{4 \rho}
$$


Moreover, we pick $N_{i} i=1,2,3$ large enough so that

$$
N_{2} \geq \frac{b j \varepsilon_{1} N_{1}+\left(\mu+\frac{n_{0}}{2}+b \varepsilon_{3}\right)}{\frac{b^{2}}{j}-\frac{b \xi}{4 \varepsilon_{2} j}-\frac{b}{j} \alpha(t)}
$$

and

$$
N_{3} \geq \frac{\left(\frac{b N_{2}}{\rho}+1\right)}{2 m_{1}} .
$$

After that, we can choose $N$ large enough such that

$$
N>\frac{1}{m_{e}}\left[\frac{b \rho N_{1}}{4 \varepsilon_{1} \mu}+N_{3} \mu_{1}+\frac{N_{2} b \mu_{1}}{4 \varepsilon_{2} \rho}-\left(\rho-\frac{\mu_{1}}{4 \varepsilon_{3}}\right)\right] .
$$

Consequently, there exists a positive constant $\eta_{1}$ such that (3.15) becomes

$$
\begin{aligned}
\frac{d}{d t} \mathcal{L}(t) \leq & -c_{1} \int_{0}^{1}\left(u_{t}^{2}+u_{x}+\varphi_{x}^{2}+\phi^{2}\right) d x+c_{2} \int_{0}^{1}\left(\phi_{t}^{2}+g^{2}\left(\phi_{t}\right)\right) d x \\
& -c_{3} \int_{0}^{1} \int_{\tau_{1}}^{\tau_{2}}\left|\mu_{2}(s)\right| z^{2}(x, 1, s, t) d s d x
\end{aligned}
$$

In this section, we state and prove our stability result.

\section{Stability Result}

Theorem 4.1. Suppose (H1), (H2), and (3.8) hold. Let $U_{0} \in \mathcal{H}$. there exist positive constants $a_{1}, a_{2}, a_{3}$ and $\eta_{0}$ such that the solution of (1.2) satisfies

$$
E(t) \leq a_{1} G_{1}^{-1}\left(a_{2} \int_{0}^{t} \alpha(s) d s+a_{3}\right), t \geq 0
$$

where

$$
G_{1}^{-1}=\int_{t}^{1} \frac{1}{G_{0}(s)} d s \text { and } G_{0}(s)=t G^{\prime}\left(\eta_{0} t\right)
$$

Remark 4.1. $G_{1}$ strictly decreases and is convex on $(0,1]$ and $\lim _{t \rightarrow 0} G_{1}(t)=+\infty$.

Proof. We multiply (3.15) by $\alpha(t)$ to get

$$
\alpha(t) \mathcal{L}^{\prime}(t) \leq-k_{1} \alpha(t) E(t)+c \alpha(t) \int_{0}^{1}\left(\phi_{t}^{2}+g^{2}\left(\phi_{t}\right)\right) d x
$$

Now, we discuss two cases:

Case I: $G$ is linear on $[0, \eta]$. In this case, using (H2) and Eq.(3.1), we deduce that

$$
\alpha(t) \mathcal{L}^{\prime}(t) \leq-k_{1} \alpha(t) E(t)+c \alpha(t) \int_{0}^{1}\left(\phi_{t}^{2}+g^{2}\left(\phi_{t}\right)\right) d x=-k_{1} \alpha(t) E(t)-c E^{\prime}(t),
$$

which can be rewritten as

$$
(\alpha(t) \mathcal{L}(t)+c E(t))^{\prime}-\alpha^{\prime}(t) \mathcal{L}(t) \leq-k_{1} \alpha(t) E(t)
$$


Using (H1), we obtain

$$
(\alpha(t) \mathcal{L}(t)+c E(t))^{\prime} \leq-k_{1} \alpha(t) E(t) .
$$

By exploiting (3.14), it can easily be shown that

$$
\mathcal{S}_{0}(t):=\alpha(t) \mathcal{L}(t)+c E(t) \sim E(t)
$$

So, for some positive constant $\lambda_{1}$, we obtain

$$
\mathcal{S}_{0}^{\prime}(t)+\lambda_{1} \alpha(t) \mathcal{S}_{0}(t) \leq 0, \forall t \geq 0
$$

The combination of Eq. (4.3) and (4.4), gives

$$
E(t) \leq E(0) e^{-\lambda_{1} \int_{0}^{t} \alpha(s) d s}=E(0) G_{1}^{-1}\left(\lambda_{1} \int_{0}^{t} \alpha(s) d s\right)
$$

Case II: $G$ is nonlinear on $[0, \eta]$. In this case, we first choose $0<\eta_{1}<\eta$ such that

$$
s g(s) \leq \min \{\eta, G(\eta)\}, \forall|s| \leq \eta_{1}
$$

Using (H2) along with fact that $g$ is continuous and $|g(s)|>0$, for $s \neq 0$, it follows that

$$
\left\{\begin{array}{l}
s^{2}+g^{2}(s) \leq G^{-1}(s g(s)), \forall|s| \leq \eta_{1} \\
c_{1}|s| \leq|s g(s)| \leq c_{2}|s|, \forall|s| \geq \eta_{1}
\end{array}\right.
$$

To estimate the last integral in Eq. (4.2), we consider the following partition of $(0,1)$ :

$$
I_{1}=\left\{x \in(0,1):\left|\phi_{t}\right| \leq \eta_{1}\right\}, I_{2}=\left\{x \in(0,1):\left|\phi_{t}\right|>\eta_{1}\right\}
$$

Now, with $I(t)$ defined by

$$
I(t)=\int_{I_{1}} \phi_{t} g\left(\phi_{t}\right) d x
$$

we have, using Jensen inequality (note that $G^{-1}$ is concave and recall (4.6))

$$
G^{-1}(I(t)) \geq c \int_{I_{1}} G^{-1}\left(\phi_{t} g\left(\phi_{t}\right)\right) d x .
$$

The combination of Eq. (4.7) and (4.8) yields

$$
\begin{aligned}
\alpha(t) \int_{0}^{1}\left(\phi_{t}^{2}+g^{2}\left(\phi_{t}\right)\right) d x & =\alpha(t) \int_{I_{1}}\left(\phi_{t}^{2}+g^{2}\left(\phi_{t}\right)\right) d x+\alpha(t) \int_{I_{2}}\left(\phi_{t}^{2}+g^{2}\left(\phi_{t}\right)\right) d x \\
& \leq \alpha(t) \int_{I_{1}} G^{-1}\left(\phi_{t} g\left(\phi_{t}\right)\right) d x+c \alpha(t) \int_{I_{2}} \phi_{t} g\left(\phi_{t}\right) d x \\
& \leq c \alpha(t) G^{-1}(I(t))-c E^{\prime}(t) .
\end{aligned}
$$

So, by substituting (4.9) into (4.2) and using (4.3) and (H1), we have

$$
\mathcal{S}_{0}^{\prime}(t) \leq-k_{1} \alpha(t) E(t)+c \alpha(t) G^{-1}(I(t))
$$


Now, for $\eta_{1}<\eta$ and $\delta_{0}>0$, using (4.10) and the fact that $E^{\prime} \leq 0, G^{\prime}>0, G^{\prime \prime}>0$ on $(0, \eta)$, we find that the functional $\mathcal{S}_{1}$, defined by

$$
\mathcal{S}_{1}(t):=G^{\prime}\left(\eta_{0} \frac{E(t)}{E(0)}\right) \mathcal{S}_{0}(t)+\delta_{0} E(t)
$$

satisfies, for some $b_{1}, b_{2}>0$,

$$
b_{1} \mathcal{S}_{1}(t) \leq E(t) \leq b_{2} \mathcal{S}_{1}(t)
$$

and

$$
\begin{aligned}
\mathcal{S}_{0}^{\prime}(t) & : \quad=\eta_{0} \frac{E^{\prime}(t)}{E(0)} G^{\prime \prime}\left(\eta_{0} \frac{E(t)}{E(0)}\right) \mathcal{S}_{0}(t)+G^{\prime}\left(\eta_{0} \frac{E(t)}{E(0)}\right) \mathcal{S}_{0}^{\prime}(t)+\delta_{0} E^{\prime}(t) \\
\leq & -k_{1} \alpha(t) E(t) G^{\prime}\left(\eta_{0} \frac{E(t)}{E(0)}\right)+c \alpha(t) G^{\prime}\left(\eta_{0} \frac{E(t)}{E(0)}\right) G^{-1}(I(t))+\delta_{0} E^{\prime}(t)
\end{aligned}
$$

Let $G^{*}$ be the convex conjugate of $G$ defined by

$$
G^{*}(s)=s\left(G^{\prime}\right)^{-1}(s)-G\left[\left(G^{\prime}\right)^{-1}(s)\right], \text { if } s \in\left(0, G^{\prime}(\eta)\right],
$$

satisfying the following general Young's inequality

$$
A B \leq G^{*}(A)+G(B), \text { if } A \in\left(0, G^{\prime}(\eta)\right], B \in(0, \eta] .
$$

With

$$
A=G^{\prime}\left(\eta_{0} \frac{E(t)}{E(0)}\right) \text { and } B=G^{-1}(I(t))
$$

using (4.6), we obtain

$$
c \alpha(t) G^{\prime}\left(\eta_{0} \frac{E(t)}{E(0)}\right) G^{-1}(I(t)) \leq c \alpha(t) G^{*}\left(G^{\prime}\left(\eta_{0} \frac{E(t)}{E(0)}\right)\right)+c \alpha(t) I(t) .
$$

By exploiting (3.1) and the fact that

$$
\begin{gathered}
G^{*}(s) \leq s\left(G^{\prime}\right)^{-1}(s) \text {, we get } \\
c \alpha(t) G^{\prime}\left(\eta_{0} \frac{E(t)}{E(0)}\right) G^{-1}(I(t)) \leq c \alpha(t) \eta_{0} \frac{E(t)}{E(0)} G^{\prime}\left(\eta_{0} \frac{E(t)}{E(0)}\right)-c E^{\prime}(t)
\end{gathered}
$$

By substituting (4.12) into Eq. (4.13), we obtain

$$
\mathcal{S}_{1}^{\prime}(t) \leq-k \alpha(t) \frac{E(t)}{E(0)} G^{\prime}\left(\eta_{0} \frac{E(t)}{E(0)}\right)=-k_{1} \alpha(t) G_{0}\left(\frac{E(t)}{E(0)}\right)
$$

where $k>0$ and $G_{0}(t)=t G^{\prime}\left(\eta_{0} t\right)$.

Note that

$$
G_{0}^{\prime}(t)=G^{\prime}\left(\eta_{0} t\right)+\eta_{0} t G^{\prime \prime}\left(\eta_{0} t\right) .
$$

So, using the strict convexity of $G$ on $(0, \eta]$, we find that $G_{0}(t), G_{0}^{\prime}(t)>0$ on $(0,1]$.With $\mathcal{S}(t):=\frac{b_{1} \mathcal{S}_{1}(t)}{E(0)}$ it is obvious that $\mathcal{S}(t) \leq \frac{E(t)}{E(0)} \leq 1$. Now, using (4.11) and (4.14), we have

$$
\mathcal{S}(t) \sim E(t)
$$


and, for some $a_{2}>0$

$$
\mathcal{S}^{\prime}(t) \leq-a_{2} \alpha(t) G_{0}(\mathcal{S}(t))
$$

Inequality (4.16) implies that

$$
\frac{d}{d t} G_{1}(\mathcal{S}(t)) \geq a_{2} \alpha(t)
$$

where

$$
G_{1}(t)=\int_{1}^{t} \frac{1}{G_{0}(s)} d s .
$$

Thus, by integrating over $[0, t]$, we obtain, for some $a_{3}>0$,

$$
\mathcal{S}(t) \leq G_{1}^{-1}\left(a_{2} \int_{0}^{t} \alpha(s) d s+a_{3}\right)
$$

Here, we used, based on the properties of $G_{0}$, the fact that $G_{1}$ is strictly decreasing on $(0,1]$.Finally, using (4.17) and (4.15), we obtain (4.1).

Acknowledgement 4.1. The author wish to thank deeply the anonymous referee for useful remarks and careful reading of the proofs presented in this paper.

Conflicts of Interest: The author(s) declare that there are no conflicts of interest regarding the publication of this paper.

\section{REFERENCES}

[1] F. Ammar-Khodja, A. Benabdallah, J.E. Muñoz Rivera, R. Racke, Energy decay for Timoshenko systems of memory type, J. Differ. Equ. 194 (2003), 82-115.

[2] T.A. Apalara, A general decay for a weakly nonlinearly damped porous system, J. Dyn. Control Syst. 25 (2019), 311-322.

[3] T.A. Apalara, General decay of solutions in one-dimensional porous-elastic system with memory, J. Math. Anal. Appl. 469 (2019), 457-471.

[4] L. Bouzettouta, S. Zitouni, Kh. Zennir, and H. Sissaoui, Well-posedness and decay of solutions to Bresse system with internal distributed delay, Int. J. Appl. Math. Stat. 56 (2017), 153-168.

[5] L. Bouzettouta. A. Djebabla, Exponential stabilization of the full von Kármán beam by a thermal effect and a frictional damping and distributed delay, J. Math. Phys. 60 (2019), 041506.

[6] L. Bouzettouta, F. Hebhoub, K. Ghennam and S. Benferdi, Exponential stability for a nonlinear Timoshenko system with distributed delay, Int. J. Anal. Appl. 19 (2021), 77-90.

[7] P.S. Casas, R. Quintanilla, Exponential stability in thermoelasticity with microtemperatures, Int. J. Eng. Sci. 43 (2005), $33-47$.

[8] A. Haraux, Nonlinear evolution equations-q-global behavior of solutions, Lecture Notes in Mathematics 841. Springer, Berlin, 1981.

[9] D. Ieşan and R. Quintanilla, On thermoelastic bodies with inner structure and microtemperatures, J. Math. Anal. Appl. 354 (2009), 12-23.

[10] H. E. Khochemane, L. Bouzettouta, A. Guerouah, Exponential decay and well-posedness for a one-dimensional porouselastic system with distributed delay, Appl. Anal. (2019), 1-15. https://doi.org/10.1080/00036811.2019.1703958. 
[11] H. E. Khochemane, A. Djebabla, S. Zitouni, L. Bouzettouta, Well-posedness and general decay of a nonlinear damping porous-elastic system with infinite memory, J. Math. Phys. 61 (2020), 021505.

[12] H. E. Khochemane, L. Bouzettouta and S. Zitouni, General decay of a nonlinear damping porous-elastic system with past history. Ann. Univ. Ferrara. 65 (2019), 249-275.

[13] I. Lasiecka, D. Tataru, Uniform boundary stabilization of semilinear wave equations with nonlinear boundary damping. Differ. Integral Equ. 6 (1993), 507-533.

[14] A. Magaña and R. Quintanilla, On the exponential decay of solutions in one-dimensional generalized porousthermoelasticity, Asymptotic Anal. 49 (2006), 183-187.

[15] S. A. Messaoudi and M. I. Mustafa, A stability result in a memory-type Timoshenko system, Dyn. Syst. Appl. 18 (2009), $457-468$.

[16] S. A. Messaoudi and A. Fareh, Exponential decay for linear damped porous thermoelastic systems with second sound, Discrete Contin. Dyn. Syst. Ser. B 20 (2015), 599-612.

[17] S. Nicaise and C. Pignotti, Stability and instability results of the wave equation with a delay term in the boundary or internal feedbacks, SIAM J. Control Optim. 45 (2006), 1561-1585.

[18] P. X. Pamplona, J. E. Muñoz Rivera and R. Quintanilla, On the decay of solutions for porous-elastic systems with history, J. Math. Anal. Appl. 379 (2011), 682-705.

[19] A. Soufyane, Energy decay for porous-thermo-elasticity systems of memory type, Appl. Anal. 87 (2008), $451-464$.

[20] A. Soufyane, M. Afilal, M. Aouam and M. Chacha, General decay of solutions of a linear one-dimensional porous thermoelasticity system with a boundary control of memory type, Nonlinear Anal., Theory Meth. Appl. 72 (2010), 3903-3910.

[21] A. Soufyane, M. Afilal and M. Chacha, Boundary stabilization of memory type for the porous-thermo-elasticity system, Abstr. Appl. Anal. 2009 (2009), Article ID 280790.

[22] S. Zitouni, L. Bouzettouta, Kh. Zennir and D. Ouchenane, Exponential decay of thermo-elastic Bresse system with distributed delay term, Hacettepe J. Math. Stat. 47 (2018), 1216-1230. 